\title{
An Automatic Number Plate Recognition of Bangladeshi Vehicles
}

\author{
Md. Ruhul Amin \\ Software Engineer \\ $1 / 4$, North Jatrabari, Dhaka-1204
}

\author{
Noor Mohammad \\ Software Engineer \\ 1/4, North Jatrabari, Dhaka-1204
}

\author{
Md. Abu Naser Bikas \\ Graduate Teaching Assistant \\ $1 / 4$, North Jatrabari, Dhaka-1204
}

\begin{abstract}
Now-a-day, the importance of automatic traffic monitoring has drawn the attention to the intelligent transport systems. A number plate is the unique identification of a vehicle. Automatic Number Plate Recognition (ANPR) is designed to locate and recognize the number plate of a moving vehicle automatically. For Bangladeshi Vehicles there are no fixed rules for number plates like other countries. Because of the variety of conditions and patterns, the recognition process of number plates for Bangladeshi vehicles is so difficult. We develop a ANPR system which is divided into three part such as number plate Localization, Extraction and Recognition. After successfully complete localization and extraction of number plate we send that portion to a Bangla OCR to fetch the text of the number plate for further processing. In this paper we present a complete ANPR system for Bangladesh Vehicles using standard algorithms with some customization and with our own algorithm for Number Plate extraction which can detect Number Plate region and successfully extract detected region and then able to recognize that number plate text.
\end{abstract}

\section{Keywords}

ANPR; Sobel Operator; Outsu threshold; Hough transform; Bangladeshi vehicles number plate

\section{INTRODUCTION}

Number Plate [1] is a rectangular, usually metal plate that bears a sequence of numbers, letters, or both and is issued by a government to identify an officially registered vehicle. It is placed front and back side of any type of vehicles. ANPR system is an image-processing technology used to identify vehicles by their license plates or number plate. They can use existing closed-circuit television or road-rule enforcement cameras, or ones specifically designed for the task. They are used by various police forces and as a method of electronic toll collection on pay-per-use roads and cataloging the movements of traffic or individuals.

ANPR system is well-known all over the world but it is comparatively very new for our country. Bangladesh is one of the most populated countries in the world and day by day the number of automobiles is increasing almost at an exponential rate. An increase in vehicles would clearly mean an increase in the number of Number Plates issued by BRTA (Bangladesh Road Transport Authority). However, not all Number Plates are veritable i.e. some Number Plates on the vehicles are made up by owners themselves. The fake Number Plates also gives freedom to an increase in vehicles related crimes. Currently, the only method to eradicate this problem is for the police to check vehicles at random and take note of the registration number and call BRTA and look up the number on an offline database [2]. After checking vehicles is free if that vehicles number plate is valid otherwise not. So in the view point of digitalization Of Bangladesh we need ANPR systems which enrich our country. There are so many effective reasons that make us interested to work in this area. Some of them are security reason, traffic controlling, toll, road monitoring, and track boarding passing vehicles. We think if such a system can be developed for our country that will definitely reduce corruption.

The remainder of the paper is organized as follows: Section 2 describes previous work related to ANPR. Section 3 describes the patterns of Bangladeshi Number plates. In section 4 we described our proposed system. From section 5 to 10 we present the steps of our developed system. Section 11 shows the experimental results. We conclude in Section 12 and with references.

\section{RELATED WORK}

The ANPR was invented in 1976 at the Police Scientific Development Branch in the UK [3]. Prototype systems were working by 1979 , and contracts were let to produce industrial systems, first at EMI Electronics, and then at Computer Recognition Systems (CRS) in Wokingham, UK. Early trial systems were deployed on the A1 road and at the Dartford Tunnel. The first arrest through detection of a stolen car was made in 1981.

There are many works done in ANPR all over the world. There have been various commercial ANPR products around the world, which include Safe-T-Cam [4], SeeCar in Israel [5], VECON in Hongkong [6], LPR in USA [7], the ANPR in UK [8], IMPS in Singapore [9], and the CARINA in Hungary[10]. Different approach can be used for developing an ANPR system. The First approaches were based on characteristics of boundary lines. The input image being first processed to enrich and enhance boundary line-information by using such algorithms as the gradient filter, and resulting in an image formed of edges. The image thus processed was converted to its binary counterpart and then processed by certain algorithms, such as Hough transform, to detect lines. Eventually, couples of 2-parallel lines were considered as a plate-designate [11] [12]. Another approach was based on the morphology of objects in an image [13] [14] [15]. This approach focuses on some salient properties of vehicle plate images such as their brightness, contrast, symmetry, angles, etc. Due to these features, this method could be used to detect the similar properties in a certain image and locate the position of number plate region. The third approach was based on statistical properties of text [16], [17]. In this approach, text regions were discovered using statistical properties of text like the variance of gray level, number of edges, edge densities in the region, etc. This approach was commonly used in finding text in images, and could well be used for discovering and designating candidate number plate areas as they include alphabets and numerals. In addition there are many approaches like artificial intelligence, genetic algorithms, mean-shift algorithm based [18] [19] [20]. 


\section{PATTERNS OF BANGLADESHI NUMBER PLATES}

In developed country there are standard rules for all the number plates for every specific country. For example in UK [21], In UK's number plate follow White front plate (to British Standard BS AU 145d), Yellow rear plate (to British Standard BS AU 145d), The registration number of the bearer vehicle in the mandatory font (black 'Charles Wright 2001'), Spacing of characters and character groups in accordance with the measurements specified in the regulations. Variations are not permitted. The plate has 7 characters in 3 parts, each with a different meaning. The first two letters show where the vehicle was registered, the local memory tag. The two numbers indicate the age of the vehicle, the age identifier. The last three letters give a unique identity to a vehicle, the random letters. But unfortunately in Bangladesh, there are no fixed rules and regulations for making number plate. For Bangladeshi vehicles it varies in number plate size, font, color etc. For this reason a complete successful ANPR is very difficult to build. Figures 1 shows different license plates of Bangladeshi vehicles and UK vehicles.

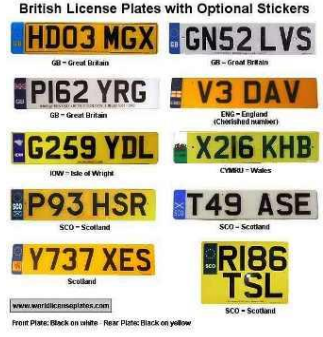

(a)

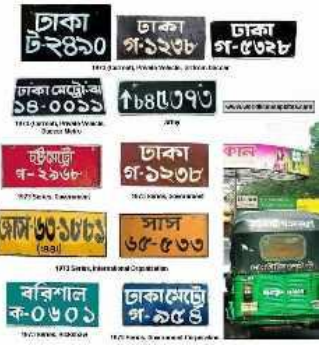

(b)
Fig 1: License Plate images.

(a) Standardized license plates of UK vehicles (b) License plates of Bangladeshi vehicles

\section{OUR DEVELOPED SYSTEM}

Number plate rules are the core concept of ANPR system. In the world which countries are using this system they have strict rules of number plate. But in Bangladesh there are no such rules. Lacking of such rules we prevent us from just using all the standard algorithm and procedure available for developing any standard ANPR system. So here we proposed and developed an ANPR system for our country for detecting and recognizing number plate of Bangladeshi vehicles. For developing our system we used the following development steps like any other number plate recognition system but with many customizations that is required for the variation of our number plated. They development steps are as follow:

* Capture Image

* Edge Detection

* Threshold

* Number Plate Localization

* Number Plate Extraction

* Number Plate Recognition

The steps are also shown in figure 2 .

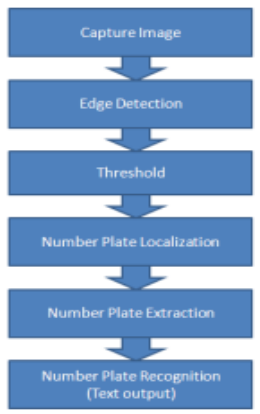

Fig 2: Diagram of the proposed license plate recognition method

To implement our system we use some well know algorithms with some customization for different steps of the development phase. We mainly focused on developing a complete ANPR system for Bangladeshi Vehicles as there does not exists any such system for Bangladesh.

In the preceding sections the steps of our developed ANPR system is briefly described.

\section{CAPTURE IMAGE}

First of all, we need clear image to locate number plate. For this purpose there are many specialized camera with high configuration which can take image of moving vehicles. But for simplicity we use Nokia 5310c Mobile to capture image. After capturing image we get an image of a vehicle like Figure3.

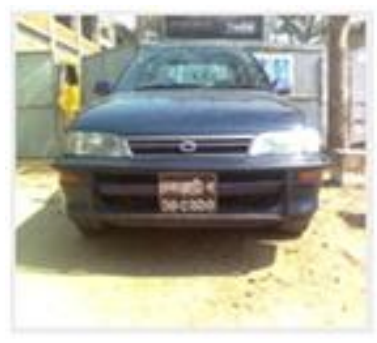

Fig 3: Captured image

\section{EDGE DETECTION}

After getting the image we need to detect the Edges of the Number Plate from the whole image. Edge detection [22] is a fundamental tool in image processing and computer vision, particularly in the areas of feature detection and feature extraction, which aim at identifying points in a digital image at which the image brightness changes sharply. The simplest Edge Detection methods rely on finding the first order differences between adjacent pixels. In our implented system we use Sobel Edge Detection [23] Operator where the operator uses two $3 \times 3$ kernels which are convolved with the original image to calculate approximations of the derivatives one for horizontal changes, and one for vertical changes.

After Edge detection the captured image is looks like Figure 4. 


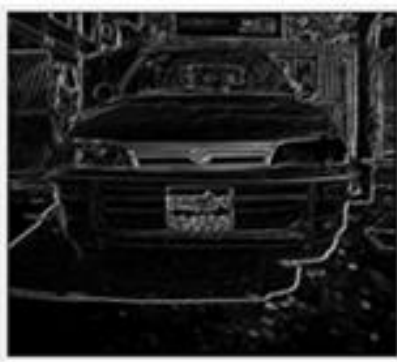

Fig 4: Image after Edge Detection

\section{THRESHOLD}

Then we need to segment the portion of our Number Plate region from the whole image. Thresholding is the simplest method of image segmentation, which involves separating an image into regions corresponding to objects. We usually try to segment regions by identifying common properties. The simplest property that pixels in a region can share is intensity. In our approach we use threshold as Otsu [24] method which stores the intensities of the pixels in an array. The threshold is calculated by using total mean and variance. It involves iterating through all the possible threshold values and calculating a measure of spread for the pixel levels each side of the threshold, i.e. the pixels that either falls in foreground or background. The aim is to find the threshold value where the sum of foreground and background spreads is at its minimum.

After threshold the image looks like as Figure 5.

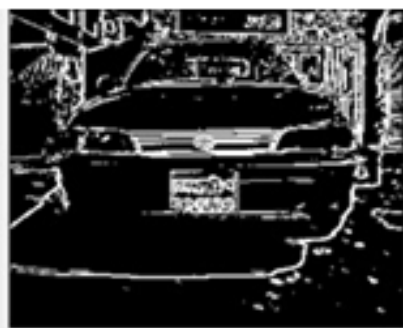

Figure 5: Binary image after thresholding

\section{NUMBER PLATE LOCALIZATION}

The next task is to localize the plate region. For localizing number plate we use the Hough transformation [25] [26] which is a feature extraction technique used to find imperfect instances of objects within a certain class of shapes by a voting procedure. This voting procedure is carried out in a parameter space, from which object candidates are obtained as local maxima in a so-called accumulator space that is explicitly constructed by the algorithm for computing the Hough transform. We are using the simplest case of Hough transform is the linear transform for detecting straight lines for our system.

We modify some portion of Hough transform for line drawing. Our modification works as follow. Hough transform gives all possible $r$ and theta corresponding one pixel. To pick those $r$ and theta, the pixel is voted by those pixels which are in the number plate region. Every pixel voted is stored in accumulator array corresponding to $r$ and theta. After that we pick $r$ and theta which has highest value. To vote in accumulator array we only calculate those theta values which range 75-105. Because of average number plate in captured image may be rotated in this range.

After localization the output image looks like Figure 6.

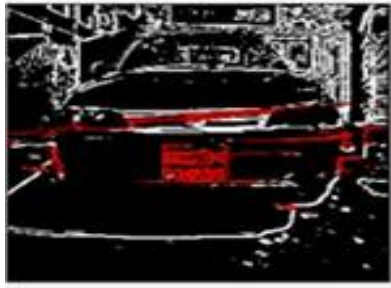

(a) (b)

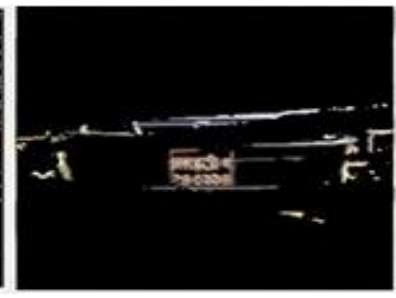

Fig 6: (a) image after line Hough (b) image after localize number plate

\section{NUMBER PLATE EXTRACTION}

This the most important part of our ANPR system. After localization of the Number Plate we need to exact only the Plate image. For this extraction purpose we used our own algorithm. The steps of our algorithm for this step works like below:

First we create an image matrix and then traverse it to get maximum feature points of rectangle shape area. In here input image has three basic color and those are red, black \& white. Our feature points are red and for that we set point 100 for that pixel value and for black and white we set respectively 30 and -50 . Then on that matrix we go to get the maximum value for rectangle shape where value is summed by rectangle area pixel values describe above. For maximum value we get a rectangle shape which gives us feature number plate region and then we crop that rectangle shape.

The pseudo code our algorithm for this step is given below:

Initialize Maximum value to zero.
Go for rectangle shape.
Calculate value for that rectangle shape and save to
current value.
Store rectangle shape if maximum value is less than the
current value
Then save that rectangle shape

After extraction the output image looks like Figure 7.

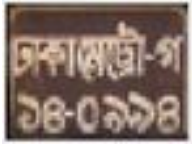

Figure 7: image after extracting number plate

\section{NUMBER PLATE RECOGNITION}

We use an OCR to recognition character from extract number plate. For Bengali language we used an open source OCR that uses Tesseract 3.0 [27] which can be trained for different character recognition. We have trained Tesseract 3.0 for Bengali character. After training Tesseract 3.0, then we use it a bangle open source OCR named SHABDAYON [28] which is developed in $\mathrm{C}++$ language. As we developed our system using java so we had to linkage java code and $\mathrm{C}++$ code. First we create a exe of OCR which is called through command line $(\mathrm{cmd})$ and give the number plate region area which one detected by our system previously and send it to OCR then it take various process and back us TEXT of the number plate. The text output is then stored our database. Then we check it is valid or invalid number plate from server.

After recognition the image through the OCR we get our text output like Figure 8. 
তাক্যা নেটো গ ১৪০৯৯8

Figure 8: Text output after recognition

\section{EXPERIMENTAL RESULTS}

We tested our system with several Bangla license plates to measure the accuracy of the system. The system is designed in JAVA (J2SE) for recognition of Bangladeshi license plates. The images for the input to the system are colored images with the size of $1200 * 1600$. The test images were taken under various illumination conditions. The results of the tests are given by Table.

Table 1: Results of Tests

\begin{tabular}{|l|c|l|}
\hline Units of ANPR System & $\begin{array}{l}\text { Number of } \\
\text { Accuracy }\end{array}$ & $\begin{array}{l}\text { Presentence of } \\
\text { Accuracy }\end{array}$ \\
\hline $\begin{array}{l}\text { Number Plate } \\
\text { Localization }\end{array}$ & $70 / 80$ & 88 \\
\hline $\begin{array}{l}\text { Number Plate } \\
\text { Extraction }\end{array}$ & $62 / 80$ & 77 \\
\hline Recognition of character & $50 / 80$ & 62 \\
\hline
\end{tabular}

It is shown that accuracy for the Localization of plate region is $88 \%, 77 \%$ for the Extraction of the plate and $62 \%$ is the percentage of accuracy of the recognition unit.

\section{CONCLUSION}

In this paper, we have developed a complete System of ANPR for Bangladeshi vehicles using standard algorithms and with some of our own algorithms for some steps. Our system is very efficient in detecting Number Plate region of any vehicles and successfully able to extract detected Number Plate from Captured image but our recognition part still has some flaws in some cases. If we can train our OCR efficiently with popular Bangla fonts then we will surely get good recognition. The main reason of this recognition problem is because of the variation of the fonts especially for hand written fonts used in our Bangladeshi vehicles. There is no fixed rule in our country for writing the number plates. If we have some fixed criteria in this case like our developed country then our system will work without any problem. Still this problem can be minimized if we can properly train the tesseract engine of OCR with lots of bangle fonts.

\section{REFERENCES}

[1] http://www.answers.com/topic/license-plate

[2] M Shadab Mashuk,M Arif Majid,Naimul Basher,Tarif Riyad Rahman, "Automatic Detection of Bangla Characters in Bangladeshi Car Registration Plates" Second International Conference on Computational Intelligence, Modelling and Simulation

[3] http://en.wikipedia.org/wiki/Automatic_number_plate_re cognition

[4] http://vision.cmit.csiro.au/project/stc/

[5] http://www.htsol.com/AboutUs.html

[6] http://www.asiavision.com.hk/

[7] http://www.perceptics.com/license-platereader.html

[8] http://www.ivsuk.com/anpr.asp

[9] http://www.singaporegateway.com/optasia/imps

[10] http://www.arhungary.hu/
[11] T. D. Duan, D. A. Duc, T. L. H. Du, "Combining Hough Transform and Contour Algorithm for detecting Vehicles License-Plates," Proceedings of 2004 International Symposium on Intelligent Multimedia, Video and Speech Processing, 2004, pp. 747-750

[12] B. Remus, "License Plate Recognition System," Proceedings of the $3^{\text {rd }}$ International Conference in Information, Communications and Signal Processing, 2001, pp. 203-206

[13] Humayun K. Sulehria, Ye Zhang, Danish Irfan, "Mathematical Morphology Methodology for Extraction of Vehicle Number Plates." International Journal of Computers, Issue 3, Volume 1, 2007

[14] R. C. Gonzalez, R. E. Woods, Digital Image Processing. 2d ed., Prentice Hall, Englewood Cliffs, NY, 2002

[15] H. L. Bai, C. P. Liu, "A Hybrid License Plate Extraction Method Based on Edge Statistics and Morphology," Proceedings of the $17^{\text {th }}$ International Conference on Pattern Recognition, 2004

[16] P. Clark, M. Mirmehdi, "Finding Text Regions using Localised Measures," Proceedings of the $11^{\text {th }}$ British Machine Vision Conference, 2000, pp. 675-684

[17] P. Clark, M. Mirmehdi, "Combining Statistical Measures to Find Image Text Regions," Proceedings of the $15^{\text {th }}$ International Conference on Pattern Recognition, 2000, pp. $450-453$

[18] Wenjing Jia, Huaifeng Zhang and Xiangjian He, "Mean Shift for Accurate Number Plate Detection" $3^{\text {rd }}$ Inernational Conference on Information Technology and Applications, July, 2005.

[19] C. M. Bishop, "Neural Networks for Pattern Recognition" Oxford: Clarendon Press, 1995

[20] R. Parisi, E. D. Di Claudio, G. Lucarelli, G. Orlandi, "Car Plate Recognition by Neural Networks and Image Processing," Proceedings of the 1998 IEEE International Symposium on Circuits and Systems, 1998, pp. 195-198

[21] http://www.regtransfers.co.uk/main/car_numberplates_re gulations.asp

[22] http://en.wikipedia.org/wiki/Edge_detection

[23] T. Venkat Narayana Rao, Dr. A. Govardhan, Syed Jahangir Badashah, "Statistical Analysis for Performance Evaluation of Image Segmentation Quality Using Edge Detection Algorithms" Int. J. Advanced Networking and Applications Volume: 03; Issue: 03; Pages:11841193(2011)

[24] http://en.wikipedia.org/wiki/Otsu\%27s_method

[25] http://en.wikipedia.org/wiki/Hough_transform

[26] Richard O. Duda,Peter E. Hart, "Use of the hough trasformtion to detect lines and curves in pictures" Published in the Comm. ACM, Vol 15, No.pp. 11-15 (January 1972)

[27] http://code.google.com/p/tesseractocr/wiki/TrainingTesseract3

[28] Farjana Yeasmin Omee, Shiam Shabbir Himel, Md. Abu Naser Bikas, "A Complete Workflow for Development of Bangla OCR", International Journal of Computer Applications (IJCA), DOI: 10.5120/2543-3483, Volume 21, Issue 9, May 2011, Pages 1-6 